\title{
Part-Based Local Shape Models for Colon Polyp Detection
}

\author{
Rahul Bhotika ${ }^{1}$, Paulo R.S. Mendonça ${ }^{1}$, Saad A. Sirohey ${ }^{2}$, Wesley D. Turner ${ }^{3}$, \\ Ying-lin Lee ${ }^{1}$, Julie M. McCoy ${ }^{1}$, Rebecca E.B. Brown ${ }^{1}$, and James V. Miller ${ }^{1}$ \\ ${ }^{1}$ GE Global Research, One Research Circle, Niskayuna, NY 12309, USA \\ \{bhotika, mendonca, leeying, mccoy, book, millerjv\}@research.ge.com \\ ${ }^{2}$ GE Healthcare, 3200 N Grandview Boulevard, Waukesha, WI, 53188, USA \\ saad.sirohey@ge.com \\ ${ }^{3}$ Kitware Inc., 28 Corporate Drive, Suite 204, Clifton Park, NY 12065, USA \\ wesley.turner@kitware.com
}

\begin{abstract}
This paper presents a model-based technique for lesion detection in colon CT scans that uses analytical shape models to map the local shape curvature at individual voxels to anatomical labels. Local intensity profiles and curvature information have been previously used for discriminating between simple geometric shapes such as spherical and cylindrical structures. This paper introduces novel analytical shape models for colon-specific anatomy, viz. folds and polyps, built by combining parts with simpler geometric shapes. The models better approximate the actual shapes of relevant anatomical structures while allowing the application of model-based analysis on the simpler model parts. All parameters are derived from the analytical models, resulting in a simple voxel labeling scheme for classifying individual voxels in a CT volume. The algorithm's performance is evaluated against expert-determined ground truth on a database of 42 scans and performance is quantified by free-response receiver-operator curves.
\end{abstract}

\section{Introduction}

According to the GLOBOCAN 2002 survey [1], colorectal cancer was the second leading cause of cancer deaths in 2002 with more than half a million deaths worldwide. The American Cancer Society estimates that 56000 people will die of the disease in the United States alone in 2005 [2]. Screening for the early detection and removal of polyps before they become cancerous is widely believed to help reduce mortality rates. However, less than half of the recommended population in the US has undergone screening, in part because of the invasive nature and risks of optical colonoscopy procedures. Virtual colonoscopy or CT colonography is emerging as an alternative, non-invasive option and may help reduce patient discomfort and thus increase compliance. Thin-slice CT scanners with their high spatial resolutions, while enabling the radiologist to see smaller structures in greater detail, pose an enormous data burden for radiologists. This data explosion motivates the need for automated systems that assist radiologists in reviewing CT colon scans with greater efficiency and improved detection sensitivity.

Several computer-aided diagnosis (CAD) systems have been developed in the last decade to find polyps [3456] in CT volumes. In general, these methods extract features such as surface curvature and intensity contrast on the colon surface to identify 
polyp-like candidates, and use a trained classifier to classify candidates. In [6], principal curvatures were used to compute a shape index at each voxel, followed by fuzzy clustering to generate candidates and classification using discriminant analysis. A support vector machine classifier trained on 3 orthogonal cross-sectional planes sampled for each candidate was used in [3]. In [7], polyp shapes were modeled using spherical harmonics and thresholds for the shape descriptors were learned from training data. In all of these methods, training and test datasets are very similar and although high performance is reported, testing is on a small number of cases, making the results inconclusive. A recent large study on 1186 scans from 3 institutions [8] evaluated the algorithm described in [5]. The method consists of extracting the surface colon wall, removing fluid if present, and segmenting candidates using surface features followed by classification. It achieved sensitivities of $89.3 \%$ at 2 false positives (FP)/case for polyps larger than $10 \mathrm{~mm}$ and $61 \%$ at $8 \mathrm{FP} /$ case for polyps above $6 \mathrm{~mm}$. Approximately onethird of the cases were used for training and the rest for testing. While the study size is impressive, results on the clinically significant medium-sized polyps $(6-9 \mathrm{~mm})$ [9] show the limitations of the learning-based CAD system.

This paper introduces a technique for highlighting polyps in CT colon scans. The algorithm is based on 3D analytical models of the local shape of colon-specific anatomical structures such as haustral folds and sessile and pedunculated polyps. Each of these structures is modeled by combining simpler shapes such as ellipsoids and tori. Principal curvatures of the implicit isosurface at each voxel are computed directly from the image function without explicitly extracting the isosurfaces. Analytical models are then used to derive constraints and decision boundaries in curvature space to label individual voxels as belonging to polyps, folds, or neither. No model fitting or training steps are required since thresholds are obtained directly from the models. Since all operations are performed locally at each voxel, the proposed method can be interpreted as a filter for highlighting relevant anatomical structures and can be easily incorporated into different viewing formats used by radiologists to read CT colon scans, such as traditional 2D slices, 3D virtual fly-throughs, or virtual dissection views.

\section{Computation of Principal Curvatures}

The curvature of the intensity isosurface passing through a voxel $\mathbf{x}$ can be computed directly from the implicit image function [10[11]. The principal curvatures $\kappa_{1}$ and $\kappa_{2}$ are independent of any transformation $I^{\prime}(\mathbf{x})=a I(\mathbf{x})+b$ applied to the image volume $I(\mathbf{x})$. In fact, $\kappa_{1}$ and $\kappa_{2}$ at a voxel $\mathbf{x}$ depend only on the shape of the associated isosurface and not the specific isovalue. Therefore only the local shapes of the relevant anatomical structures has to be considered, not the exact intensity profiles.

\section{Shape Models for Colon Structures}

Given the principal curvatures at a voxel, analytical models for the shapes of relevant anatomical structures can be used to set thresholds and classify the local shape at a voxel as ellipsoidal or toroidal [11]. Two major contributions of this work are in building such analytical models for colon-specific anatomy and addressing more complicated structures by combining simpler model parts. 

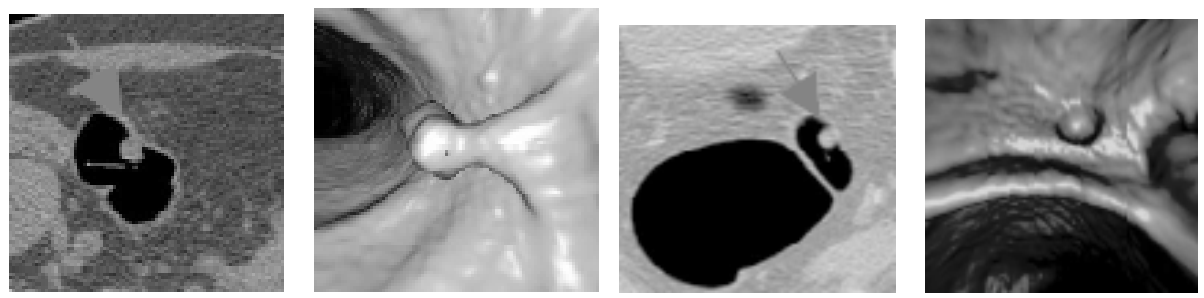

Fig. 1. Colon structures of interest From left to right: 2D and 3D rendered views of a typical pedunculated polyp, and 2D and 3D views of a typical sessile polyp. The 3D views also show haustral folds running across the colon cross-section.

After bowel cleansing and insufflation of the colon, the colon wall will typically display a circular cross-section and for all purposes can be considered locally flat compared to the attached structures. The normal colon also has haustral folds produced by the shortening of colon segments which form ridge-like structures running circumferentially, as seen in Fig. 1. Colonic polyps, on the other hand, are protrusions from the wall of the colon into the lumen and generally fall into three categories based on shape: pedunculated, sessile, and flat. This work introduces analytical models haustral folds and for pedunculated and sessile polyp lesions.

\subsection{Pedunculated Polyps}

Pedunculated polyps are mushroom-like capped structures attached by a thin stalk to the colon wall, as shown in Fig. 1. The shape of the polyp cap is locally ellipsoidal and therefore well-approximated by the ellipsoidal patches used for the nodule model in [11], without having to explicitly model the stalk.

\subsection{Sessile Polyp Model}

Sessile polyps are relatively flat polyps with a broad base and no stalk. This configuration can be geometrically modeled by combining a half ellipsoid with a base radius $c$ and height $a$, and a semi-toroidal rim with radius $r$, as depicted in Fig.2(a). The analysis of the local shape of sessile polyps can be carried out independently on the individual ellipsoidal and toroidal parts and then combined as shown below.

The ellipsoidal part of the sessile polyp, $\mathscr{M}_{\mathrm{SP}, \mathrm{E}}$, can be parameterized as

$$
\begin{aligned}
\mathscr{M}_{\mathrm{SP}, \mathrm{E}}: \Pi \times \Theta \times \Phi & \rightarrow \mathbb{R}^{3} \\
(\rho, \theta, \phi) & \mapsto \mathbf{x}=\left[\begin{array}{c}
\rho c \cos \theta \cos \phi \\
\rho c \sin \theta \cos \phi \\
\rho(r+a \sin \phi)
\end{array}\right]
\end{aligned}
$$

where $\Pi=[0,1], \Theta=[0,2 \pi), \Phi=[0, \pi / 2]$, with each choice of $\rho \in \Pi$ defining a different isosurface within the volume of the sessile polyp model. The model parameters $\mathbf{m}_{\mathrm{SP}}=(a, c, r)$ determine the shape of the isosurfaces. A cross-sectional side-view of the outermost isosurface of the sessile polyp model is shown in Fig. 2(b). The model parameters $\mathbf{m}_{\mathrm{SP}}$ lie in the domain $\mathbf{M}_{\mathrm{SP}}=\left\{(a, c, r) \in \mathbb{R}^{3} \mid 0<a \leq c, 0<r\right\}$. 


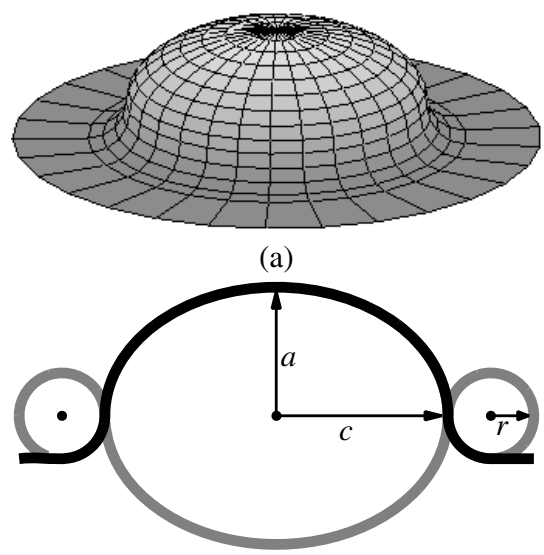

(b)

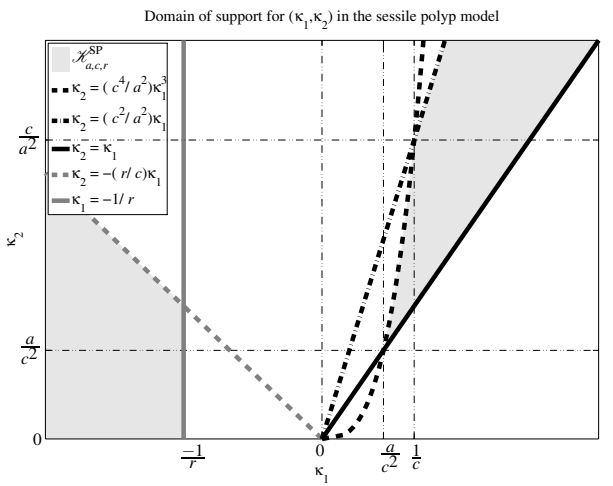

(c)

Fig. 2. Sessile Polyp Model (a) Three-dimensional representation of the sessile polyp. (b) Parameters of the sessile polyp model: the ellipsoidal cap of the sessile polyp has semi-axis $a \leq b=c$, and the toroidal contact has an inner radius of $r$. The full surface can be obtained by rotating the dark curve around the $\boldsymbol{a}$ direction. (c) Shaded region shows support of the sessile polyp model in $\boldsymbol{\kappa}$ space.

Let $E(\mathbf{x})=\frac{x^{2}}{c^{2}}+\frac{y^{2}}{c^{2}}+\frac{z^{2}}{a^{2}}$. The equation $E(\mathbf{x})=\rho$ defines an isosurface. Solving for $\boldsymbol{\kappa}=\left(\kappa_{1}, \kappa_{2}\right)$ and transforming into polar coordinates yields

$$
\kappa_{1}=\frac{a}{\rho c\left(a^{2}-\left(a^{2}-c^{2}\right) \sin ^{2} \phi\right)^{1 / 2}} \text { and } \kappa_{2}=\frac{a c}{\rho\left(a^{2}-\left(a^{2}-c^{2}\right) \sin ^{2} \phi\right)^{3 / 2}} .
$$

Enforcing simple constraints such as $0 \leq \kappa_{1} \leq \kappa_{2}, 0 \leq \sin \phi \leq 1$ with $\phi \in[0, \pi / 2]$, and $0 \leq \rho \leq 1$, one can determine the region of support $\mathscr{K}_{a, c}^{S P, E}$ for $\mathscr{M}_{\text {SP,E }}$ in $\boldsymbol{\kappa}$ space where (2) holds, as shown in Fig.2(c). The pedunculated polyp model has a very similar region of support (not shown).

The toroidal rim (or "neck") of the sessile polyp, $\mathscr{M}_{\mathrm{SP}, \mathrm{T}}$, can similarly be parameterized as

$$
\begin{aligned}
\mathscr{M}_{\mathrm{SP}, \mathrm{T}}: \Pi \times \Theta \times \Phi & \rightarrow \mathbb{R}^{3} \\
(\rho, \theta, \phi) & \mapsto \mathbf{x}=\left[\begin{array}{c}
\rho(c+r-r \cos \phi) \cos \theta \\
\rho(c+r-r \cos \phi) \sin \theta \\
\rho(r+r \sin \phi)
\end{array}\right]
\end{aligned}
$$

where $\Pi=[0,1], \Theta=[0,2 \pi), \Phi=[-\pi / 2,0)$. It can be shown that the principal curvatures at an isosurface level $\rho \in \Pi$ for $\mathscr{M}_{\mathrm{SP}, \mathrm{T}}$ are given by

$$
\kappa_{1}=-\frac{1}{\rho r} \text { and } \kappa_{2}=\frac{\cos \phi}{\rho(c+r(1-\cos \phi))} .
$$

The bounds for the region of support $\mathscr{K}_{a, c, r}^{S P, T}$ in $\boldsymbol{\kappa}$ space where (4) holds can also be derived as before. The combined support region for the sessile polyp model is given by the domain $\mathscr{K}_{a, c, r}^{\mathrm{SP}}=\mathscr{K}_{a, c}^{S P, E} \cup \mathscr{K}_{a, c, r}^{S P, T}$ and is shown in Fig.2(2). 


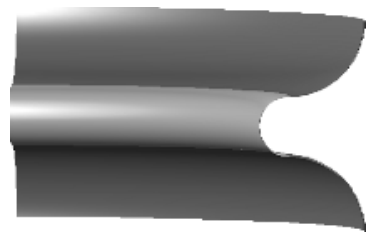

(a)

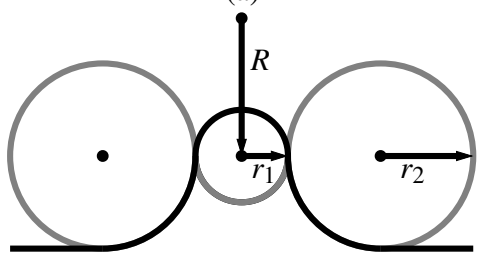

(b)

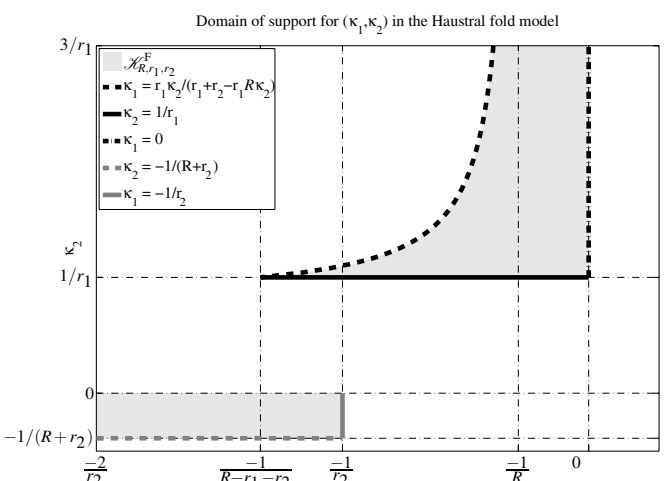

(c)

Fig. 3. Haustral Fold model (a) Three-dimensional representation of the haustral fold with toroidal ridge and neck parts (b) Parameters of the fold model: the torus representing the fold ridge has radius $r_{1}$, and the toroidal contacts forming the neck each of radius $r_{2}$. All three tori have large radius $R$. The full surface is obtained by rotating the black curve along a direction parallel to the colon centerline. (c) Shaded region shows support of the fold model in $\boldsymbol{\kappa}$ space.

\subsection{Haustral Fold Model}

Following a procedure similar to that for sessile polyps, haustral folds can be modeled by combining three different tori, one with radius $r_{1}$ for the ridge and one each on either side of the ridge with radius $r_{2}$, to model the neck connecting the fold ridge and the colon wall, as depicted in Fig. 3 (a). The fold curves around the colon wall, rotating with a radius $R$ about the colon centerline. The model parameters $\mathbf{m}_{\mathrm{F}}=\left(R, r_{1}, r_{2}\right)$ determine the shape of the isosurfaces of the fold and lie in the domain $\mathbf{M}_{\mathrm{F}}=\left\{\left(R, r_{1}, r_{2}\right) \in \mathbb{R}^{3} \mid 0<\right.$ $\left.r_{1} \leq r_{2}, 0<R\right\}$. A cross-sectional side-view of the outermost isosurface of the haustral fold model is shown in Fig. 3 b). The torus representing the neck of the fold can be parameterized as

$$
\begin{aligned}
\mathscr{M}_{\mathrm{F}, \mathrm{R}}: \Pi \times \Theta \times \Phi & \rightarrow \mathbb{R}^{3} \\
(\rho, \theta, \phi) & \mapsto \mathbf{x}=\left[\begin{array}{c}
\left(R-\rho r_{2}-\rho r_{1} \sin \phi\right) \cos \theta \\
\left(R-\rho r_{2}-\rho r_{1} \sin \phi\right) \sin \theta \\
\rho r_{1} \cos \phi
\end{array}\right]
\end{aligned}
$$

where $\Pi=[0,1], \Theta=\left[0, \theta_{0}\right], \Phi=(0, \pi / 2]$, with $\rho$ representing the different levels of the model isosurfaces and $\theta$ and $\phi$ representing the location on a particular isosurface. Solving at a particular isosurface level $\rho$ for $\boldsymbol{\kappa}=\left(\kappa_{1}, \kappa_{2}\right)$ and transforming into polar coordinates using the parameterization in (5) yields

$$
\kappa_{1}=\frac{-\sin \phi}{R-\rho r_{2}-\rho r_{1} \sin \phi} \text { and } \kappa_{2}=\frac{1}{\rho r_{1}} .
$$

Similarly the neck tori each follow the parameterization give by

$$
\begin{aligned}
\mathscr{M}_{\mathrm{F}, \mathrm{N}}: \Pi \times \Theta \times \Phi & \rightarrow \mathbb{R}^{3} \\
(\rho, \theta, \phi) & \mapsto \mathbf{x}=\left[\begin{array}{c}
\left(R-\rho r_{2}(1+\sin \phi)\right) \cos \theta \\
\left(R-\rho r_{2}(1+\sin \phi)\right) \sin \theta \\
\rho\left(r_{1}+r_{2}(1-\cos \phi)\right)
\end{array}\right]
\end{aligned}
$$


with $\Pi=[0,1], \Theta=\left[0, \theta_{0}\right], \Phi=[-\pi / 2,0]$. The torus on the other side of the fold is exactly the same except $\Phi=[\pi, 3 \pi / 2]$.

The principal curvatures for the fold neck are given by

$$
\kappa_{1}=\frac{-1}{\rho r_{2}} \text { and } \kappa_{2}=\frac{\sin \phi}{R-\rho r_{2}(1+\sin \phi)} .
$$

The bounds for the regions of support $\mathscr{K}_{R, r_{1}, r_{2}}^{F, R}$ and $\mathscr{K}_{R, r_{1}, r_{2}}^{F, N}$ in $\boldsymbol{\kappa}$ space where (6) and (8) are respectively valid for the fold ridge and neck can also be extracted using model constraints as was done for the sessile polyp. The combined support region for the fold model is given by the domain $\mathscr{K}_{R, r_{1}, r_{2}}^{\mathrm{F}}=\mathscr{K}_{R, r_{1}, r_{2}}^{F, R} \cup \mathscr{K}_{R, r_{1}, r_{2}}^{F, N}$, and is shown in Fig. 3 (c).

\section{Implementation and Experimental Results}

Implementation The algorithm was implemented using the open source Insight Toolkit (ITK) [12]. The volume image was smoothed by convolution with a Gaussian kernel to reduce the effect of noise. The colon volume was automatically extracted as follows. All voxels in the CT volume were labeled as non-body or body using an adaptive threshold between air and body tissue. Running connected components on the non-body voxels yielded a set of air regions. Frequently, colon CT scans also contain the lower part of the lungs close to the diaphragm. Regions outside the body or in the lung were discarded automatically, leaving only air-filled regions of the colon. The air-filled regions were dilated and then subtracted from the dilated regions, giving a thin layer of voxels consisting of just the colon wall and attached structures. All subsequent processing was restricted to this region-of-interest (ROI). The colon segmentation assumes that the colon was prepared through cleansing and insufflation.

The principal curvatures at every voxel in the ROI were computed as described in [1011]. The pedunculated and sessile polyp models and the haustral fold model provide regions of support in $\boldsymbol{\kappa}$ space. Given principal curvatures computed at a voxel $\mathbf{x}$, it was labeled as belonging to a polyp or a fold or neither, depending on where the $\kappa_{1}(\mathbf{x})$ and $\kappa_{2}(\mathbf{x})$ values lied in $\boldsymbol{\kappa}$ space. The decision boundaries depend on the model parameters $\mathscr{K}_{a, c, r}^{\mathrm{SP}}$ and $\mathscr{K}_{R, r_{1}, r_{2}}^{\mathrm{F}}$ and were chosen either based on representative values for clinically relevant target lesions or knowledge of anatomical structures. For example, the radius $R$ in the fold model was approximated by the radius of the colon cross-section. The fact that all model parameters can be specified in physical units and the ITK-based implementation can maintain and process data in physical space allowed all model constraints to be easily propagated throughout the algorithm.

Since the curvature computations are susceptible to noise and the analytical models are idealized, morphological operations were applied on the polyp and fold responses to remove spurious small polyp responses occurring in isolation or in small pockets within large fold response regions. These final polyp and fold responses can be presented to radiologists as overlays on top of the the original $\mathrm{CT}$ volume, either on the $2 \mathrm{D}$ axial slices or on a 3D rendering as shown in Fig. 4(left). The algorithm takes about 30 seconds to run on a $3 \mathrm{GHz}$ Xeon processor with $2 \mathrm{~GB}$ RAM.

Ground Truth and Validation. To quantify the algorithm's performance, neighboring voxel responses were grouped and counted as a single detection. The validation dataset 

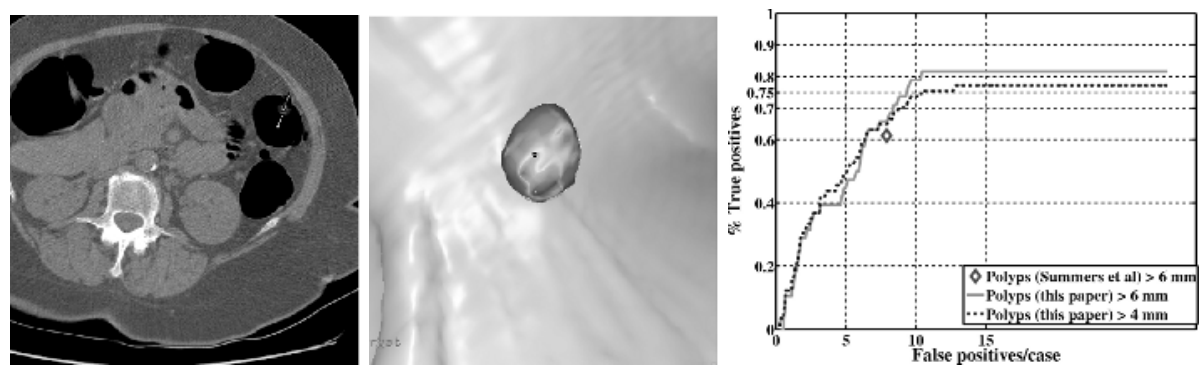

Fig. 4. Algorithm results The two images on the left show polyp responses generated by the algorithm overlaid on a 2D CT slice and a 3D rendering, respectively. On the right are FROC curves showing the algorithm's performance with ground truths containing polyps $>6 \mathrm{~mm}$ (light, solid curve) and polyps $>4 \mathrm{~mm}$ (dark, dashed curve).

Table 1. Comparison of proposed method with CAD evaluation from [8]: The voxel-labeling scheme proposed in this paper does not require any training and compares favorably in running time and performance, though it has been validated on a much smaller dataset

\begin{tabular}{|c|c|c|c|c|c|}
\hline Algorithm & $\begin{array}{c}\text { Training } \\
\text { cases }\end{array}$ & $\begin{array}{c}\text { Testing } \\
\text { cases }\end{array}$ & $\begin{array}{c}\text { Sensitivity @ FP } \\
\text { polyps > 6 mm }\end{array}$ & $\begin{array}{c}\text { Sensitivity @ FP } \\
\text { polyps > 4 mm }\end{array}$ & $\begin{array}{c}\text { Running } \\
\text { time/case }\end{array}$ \\
\hline Proposed method & 0 & 42 & $31 / 38=81.6 \% @ 10.4$ & $43 / 57=75.4 \%$ @ 10.4 & $30 \mathrm{sec}$ \\
\hline Summers et al [8] & 394 & 792 & $73 / 119=61.3 \%$ @ 8.0 & - & $20 \mathrm{~min}$ \\
\hline
\end{tabular}

consisted of $42 \mathrm{CT}$ scans. A low-dose screening protocol with $120 \mathrm{mAs}, 120 \mathrm{kVp}$, and $0.6 \mathrm{~mm}$ slice spacing was used. Ground truth annotations including polyp location and diameter were provided by an expert radiologist. Current screening protocols consider all polyps above $6 \mathrm{~mm}$ in diameter as clinically relevant. The algorithm achieved a sensitivity of $81.6 \%$ at $10.4 \mathrm{FP}$ or $76.3 \%$ at $6.2 \mathrm{FP} /$ case for the 38 polyps in the ground truth with diameter above $6 \mathrm{~mm}$. The complete free-response receiver operator curve (FROC) is shown in Fig. 4 (right). Radiologists currently do not report findings of "diminutive" polyps (between 4-6 mm in diameter) [9]. However, a follow-up repeat screening in 5 years is generally recommended in such cases. Therefore, the algorithm was also evaluated against all polyps above $4 \mathrm{~mm}$ in the ground truth (57 polyps) and achieved a sensitivity of $77.2 \%$ at $12.9 \mathrm{FP} /$ case or $75.4 \%$ at $10.4 \mathrm{FP} /$ case, as shown in Fig. 4 (right).

It is difficult to perform a fair comparison of CAD methods because of differences in test databases and validation techniques. Also, very few CAD evaluations on large databases are available. Recently, a study evaluating a classifier-based CAD algorithm on an 1186 cases from 3 institutions ( $1 \mathrm{~mm}$ slice spacing $100 \mathrm{mAs}, 120 \mathrm{kVp}$ ) has been published [8]. Table 1 attempts to compares the method proposed here to the testing infrastructure and the results in this study. The relatively lower performance of the learning-based CAD algorithm used in [8] shows that the model-based approach presented in this paper has promise although it has been tested on a much smaller database. 


\section{Conclusion}

This paper introduces a novel voxel labeling scheme for highlighting lesions in CT colon scans. Geometric models of colon-specific anatomical structures, namely pedunculated or sessile polyps and haustral folds were developed by combining ellipsoids and tori. Constraints derived from the models are used to classify each voxel based on the principal curvatures computed at that voxel. The method requires neither segmentation of the relevant anatomical structures nor sophisticated classifiers trained over a set of image features. It can be viewed as a simple, fast filter that highlights voxels belonging to specific anatomical shapes and can aid the radiologist in reviewing CT colon exams with greater efficiency and sensitivity. Validation results show the promise of the model-based approach taken in this paper. Future work includes modeling flat polyps and extending the models to screening protocols with different patient preparations.

\section{References}

1. Ferlay, J., Bray, F., Pisani, P., Parkin., D.M.: GLOBOCAN 2002: Cancer incidence, mortality and prevalence worldwide. Technical report, IARC CancerBase No. 5. version 2.0, IARCPress, Lyon, France (2004) http://www-dep.iarc.fr/.

2. American Cancer Society: Colorectal cancer: Facts and figures, special edition (2005)

3. Göktürk, S.B., Tomasi, C., Burak, A., Beaulieu, C.F., Paik, D.S., Brooke Jeffrey Jr., R., Yee, J., Napel, S.: A statistical 3-D pattern processing method for computer-aided detectin of polyps in CT colonography. IEEE Trans. Medical Imaging 20(12) (2001) 1251-1260

4. Paik, D.S., Beaulieu, C.F., Rubin, G.D., Acar, B., Jeffrey, Jr., R.B., Yee, J., Dey, J., Napel, S.: Surface normal overlap: A computer-aided detection algorithm with application to colonic polyps and lung nodules in helical CT. IEEE Trans. Medical Imaging 23(6) (2004) 661-675

5. Summers, R.M., Johnson, C.D., Pusanik, L.M., D., M.J., Youssef, A.M., Reed, J.E.: Automated polyp detection at CT colonography: Feasibility assessment in a human population. Radiology 219 (2001) 51-59

6. Yoshida, H., Näppi, J.: Three-dimensional computer-aided diagnosis scheme for detection of colonic polyps. IEEE Trans. Medical Imaging 20(12) (2001) 1261-1274

7. Kiss, G., Van Cleynenbreugel, J., Drisis, S., Bielen, D., Marchal, G., Suetens, P.: Computer aided detection for low-dose CT colonography. In: Medical Image Computing and Computer-Assisted Intervention. Number 3749 in Lecture Notes in Computer Science, Springer-Verlag (2005) 859-867

8. Summers, R.M., Yao, J., et al, P.P.J.: Computed tomographic virtual colonoscopy computeraided polyp detection in a screening population. Gastroenterology 129 (2005) 1832-1844

9. Pickhardt, P.: Target lesion: The radiologist's perspective. In: Sixth International Symposium on Virtual Colonoscopy, Boston, MA (2005) 60-62

10. Vos, F.M., Serlie, I.W.O., van Gelder, R.E., Post, F.H., Truyen, R., Gerritsen, F.A., Stoker, J., Vossepoel, A.M.: A new visualization method for virtual colonoscopy. In Niessen, W.J., Viergever, M.A., eds.: Medical Image Computing and Computer-Assisted Intervention. Number 2208 in Lecture Notes in Computer Science, Berlin, Springer-Verlag (2001) 645-654

11. Mendonça, P.R.S., Bhotika, R., Sirohey, S., Turner, W.D., Miller, J.V., Avila, R.S.: Modelbased analysis of local shape for lesion detection in CT scans. In Duncan, J., Gerig, G., eds.: Medical Image Computing and Computer-Assisted Intervention. Number 3749 in Lecture Notes in Computer Science, Palm Springs, CA, USA, Springer-Verlag (2005) 688-695

12. Ibáñez, L., Schroeder, W., Ng, L., Cates, J.: The ITK Software Guide. Kitware Inc. (2003) 